\title{
THE STATIONARY SET OF A GROUP ACTION
}

\author{
DENNIS STOWE
}

\begin{abstract}
If a point is stationary for a differentiable group action, M. Hirsch suggested that the vanishing of the first group cohomology at that point might imply stability of the point under perturbations of the action. This is proved for compactly generated Lie groups. Further implications of the cohomology condition are also proved.
\end{abstract}

This paper concerns the question: given an action of a topological group on a manifold and a point which is stationary for this action, what conditions will insure that the point is stable? It is possible that a good general answer to this question would include conditions on the global topology of the manifold. This paper, however, presents conditions on just the linear part of the action at the stationary point which are sufficient for its stability, and which in fact are sufficient for the stability of the stationary set near that point.

Let $G$ be a topological group and $M$ a finite-dimensional $C^{1}$ manifold. An action of $G$ on $M$ is a continuous homomorphism $\alpha: G \rightarrow \operatorname{Diff}^{1}(M)$. Here $\operatorname{Diff}^{1}(M)$, the group of $C^{1}$ diffeomorphisms of $M$, is understood to carry the weak topology, in which convergence of a net of diffeomorphisms means uniform convergence on compact sets of the corresponding homeomorphisms of $T M$. The set of all actions of $G$ on $M$ itself has a topology, namely the compact-open topology which it inherits as a subset of the set of continuous maps from $G$ to $\operatorname{Diff}^{1}(M)$. A point of $M$ is stationary for $\alpha$ if it is fixed by $\alpha(g)$ for every $g \in G$. Finally, a stationary point $p$ is stable if, given any neighborhood $U$ of $p$, there is a neighborhood $N$ of $\alpha$ such that each $\beta \in N$ has a stationary point in $U$.

An action with stationary point $p$ induces a linear action (that is, an action for which each element of $G$ acts by a linear map) on the tangent space $M_{p}$, given by $g \mapsto D \alpha(g)_{p}$. This linear action is the focus of this paper, and I begin by looking at a general linear action $\gamma: G \rightarrow \mathrm{GL}(E)$, where $E$ is a finite-dimensional vector space. For $u: G \rightarrow E$, a map $\gamma_{u}: G \rightarrow \operatorname{Aff}(E)$ is defined by $\gamma_{u}(g) x=\gamma(g) x+u(g) \cdot \gamma_{u}$ will be an action exactly when

(1) $u$ is continuous and

(2) $u(g h)=\gamma(g) u(h)+u(g)$ for all $g, h \in G$.

A point $y \in E$ will be stationary for $\gamma_{u}$ exactly when

(3) $u(g)=y-\gamma(g) y$ for all $g \in G$.

Maps satisfying (1) and (2) ("crossed homomorphisms") form a vector space; the

Received by the editors May 23, 1979.

AMS (MOS) subject classifications (1970). Primary 54H15, 57E20; Secondary 58F10.

Key words and phrases. Topological group, group action, stability, semisimple Lie group, group cohomology. 
totality of maps given by (3) as $y$ ranges over $E$ form a subspace. Their quotient is written as $H^{1}(G, E)$, where $\gamma$ is understood [8]. M. Hirsch showed (Theorem 1.1 of [5]) that in order for the origin to be stable for $\gamma$, it is necessary that $H^{1}(G, E)=0$. In another paper [4], he conjectured a strong relationship between the stability of a stationary point $p$ of an arbitrary action and $H^{1}\left(G, M_{p}\right)$ being trivial. Two examples show the limitations of this relationship. The first is the action of $\mathbf{Z}$ on $\mathbf{R}$ generated by the diffeomorphism $x \mapsto x+x^{3}$. The origin is a stable stationary point, but $H^{1}$ is not trivial for the linear part there. In the second example, the acting group is the free abelian group, with the discrete topology, on infinitely many generators $g_{1}, g_{2}, \ldots$ Let $\alpha$ be an action of this group on $\mathbf{R}$ such that $\alpha\left(g_{1}\right)$ has $\mathrm{Z}$ as its fixed point set, with 0 hyperbolic, and $\alpha\left(g_{i}\right)=\mathrm{id}_{R}$ for $i>2$. Then $H^{1}$ is trivial for the linear part of this action at 0 , but 0 is not stable.

1. A criterion for stability. For two common classes of groups, triviality of $\boldsymbol{H}^{1}$ does imply stability.

THEOREM A. If $G$ is a finitely-generated discrete group and $H^{1}\left(G, M_{p}\right)=0$, then $p$ is stable.

THEOREM B. If $G$ is a connected Lie group and $H^{1}\left(G, M_{p}\right)=0$, then $p$ is stable.

These results together with those of Hirsch provide a necessary and sufficient condition for the stability of the origin under perturbations of linear actions of such groups.

I first prove Theorems $\mathrm{A}$ and $\mathrm{B}$ under the assumption that the manifold is $\mathbf{R}^{\boldsymbol{n}}$. Both proofs rely heavily on the following light lemma.

Lemma. If $A \in \operatorname{Hom}\left(\mathbf{R}^{n}, \mathbf{R}^{n}\right), K$ is a convex subset of $\mathbf{R}^{n}$ and $f: \mathbf{R}^{n} \rightarrow \mathbf{R}^{n}$ satisfies $\left\|D f_{x}-A\right\| \leqslant \xi$ for $x \in K$, then $|f(a)-f(b)-A(a-b)|<\xi|a-b|$ for all $a, b \in$ K.

Proof of Theorem A. Let $\alpha$ be an action of $G$ on $\mathbf{R}^{n}$ having the origin as stationary point and $H^{1}=0$ for the linear part $\gamma$ there. Let $g_{1}, \ldots, g_{k}$ be generators of $G$,

$$
V=\bigcap_{g \in G} \operatorname{ker}(\gamma(g)-I)=\operatorname{ker}\left(\gamma\left(g_{1}\right)-I\right) \cap \cdots \cap \operatorname{ker}\left(\gamma\left(g_{k}\right)-I\right)
$$

and $W$ be a subspace complementary to $V$. For $x \in \mathbf{R}^{n}$, let

$$
h(x)=\sum_{j=1}^{k}\left|\alpha\left(g_{j}\right) x-x\right|^{2}
$$

the origin is a strict local minimum of $\left.h\right|_{W}$. I assume the origin not to be stable and show that this leads to a contradiction. Under this assumption, there are an open ball $B$ around the origin and actions $\beta_{1}, \beta_{2}, \ldots$ such that

(1) $h$ is positive on $W \cap \operatorname{clos}(B)$ except at the origin.

(2) For $j=1, \ldots, k, \beta_{i}\left(g_{j}\right) \rightarrow \alpha\left(g_{j}\right)$ and $D \beta_{i}\left(g_{j}\right) \rightarrow D \alpha\left(g_{j}\right)$ uniformly on $B$.

(3) If $h_{i}(x)$ is defined to be $\sum_{j=1}^{k}\left|\beta_{i}\left(g_{j}\right) x-x\right|^{2}$, then $\left.h_{i}\right|_{W \cap \cos (B)}$ has a positive minimum $x_{i} \in W \cap B$. 
A result of these conditions is that $x_{i} \rightarrow 0$. Therefore $\beta_{i}(g) x_{i} \rightarrow 0$ for each $g \in G$, for this is true of the identity element of $G$, and by (2) the set of elements for which it holds is closed under left multiplication by $g_{1}, \ldots, g_{k}$. Similarly, for every pair $g, h \in G$, the supremum of $\left\|D \beta_{i}(g)_{x}-\gamma(g)\right\|$ along the segment from $x_{i}$ to $\beta_{i}(h) x_{i}$ tends to zero as $i$ tends to infinity. By the lemma, $\beta_{i}(g) \beta_{i}(h) x_{i}-\beta_{i}(g) x_{i}$ is within $\xi\left|\beta_{i}(h) x_{i}-x_{i}\right|$ of $\gamma(g)\left(\beta_{i}(h) x_{i}-x_{i}\right)$, where $\xi$ is this supremum. For $f \in G$, let $u(f)=\lim _{i \rightarrow \infty}\left(h_{i}\left(x_{i}\right)\right)^{-1 / 2}\left(\beta_{i}(f) x_{i}-x_{i}\right)$ provided this limit exists. Then if $u(g)$ and $u(h)$ exist,

$$
u(g h)=\lim _{i \rightarrow \infty} \frac{\beta_{i}(g h) x_{i}-x_{i}}{\left(h_{i}\left(x_{i}\right)\right)^{1 / 2}}=\lim _{i \rightarrow \infty}\left[\frac{\beta_{i}(g) \beta_{i}(h) x_{i}-\beta_{i}(g) x_{i}}{\left(h_{i}\left(x_{i}\right)\right)^{1 / 2}}+\frac{\beta_{i}(g) x_{i}-x_{i}}{\left(h_{i}\left(x_{i}\right)\right)^{1 / 2}}\right]
$$

exists and satisfies $u(g h)=\gamma(g) u(h)+u(g)$. We may pass to a subsequence and assume that $\left(h_{i}\left(x_{i}\right)\right)^{-1 / 2}\left(\beta_{i}\left(g_{1}\right) x_{i}-x_{i}, \ldots, \beta_{i}\left(g_{k}\right) x_{i}-x_{i}\right)$ converges in $S^{n k-1}$. Then $u(g)$ exists for all $g \in G$. $u$ is then a nonzero crossed homomorphism. Since $H^{1}=0$, there is some $y \in \mathbf{R}^{n}$, which clearly may be chosen from $W$, with $u(g)=y-\gamma(g) y$ for all $g \in G$. The derivative of $h_{i}$ at $x_{i}$ in the direction of $y$ is $2 \sum_{j=1}^{k}\left(\beta_{i}\left(g_{j}\right) x_{i}-x_{i}\right) \cdot\left(D \beta_{i}\left(g_{j}\right)_{x_{i}} y-y\right)$. Since this quantity divided by $\left(h_{i}\left(x_{i}\right)\right)^{1 / 2}$ tends to $2 \sum_{j=1}^{k} u\left(g_{j}\right) \cdot\left(-u\left(g_{j}\right)\right)=-2$, it is negative for large enough $i$. This contradicts the fact that $x_{i}$ is a minimum of $\left.h_{i}\right|_{W \cap B}$. The origin is therefore stable for $\alpha$. Q. E. D.

It has been pointed out to me that $\mathrm{W}$. Thurston's development of approximate cocycles in [9] is quite like the preceding argument.

The proof of Theorem B is similar, but the following is needed to establish continuity of the crossed homomorphism $\left(r^{\prime}\right.$ denotes $\left.d r / d t\right)$.

Lemma. Let $A$ be an $n \times n$ matrix and $\varphi: \mathbf{R} \times \mathbf{R}^{n} \rightarrow \mathbf{R}^{n}$ a $C^{1}$ flow, written $\varphi_{t} x$. Suppose that for all $t \in[0,1],\left\|\left(D \varphi_{t}\right)_{0}-\exp (t A)\right\|<\xi<1 / 2$. Then, letting $r(t)=$ $\varphi_{t} 0,\left|r(t)-r(0)-\left(\int_{0}^{t} \exp (s A) d s\right) r^{\prime}(0)\right| \leqslant \xi t\left|r^{\prime}(0)\right|$ for all $t \in[0,1]$. This implies that if $a \in(0,1]$ is small enough that the matrix $C=\int_{0}^{a} \exp (s A) d s$ is invertible and $\left\|C^{-1}\right\|<2 / a$, then for $t \in[0, a]$,

$$
\left|r(t)-r(0)-\left(\int_{0}^{t} \exp (s A) d s\right) C^{-1}(r(a)-r(0))\right| \leqslant \xi K|r(a)-r(0)|,
$$

where $K$ is independent of $t$ and $\xi$.

Proof. The derivative of $\varphi_{s}$ at 0 in the direction of $r^{\prime}(0)$ is $r^{\prime}(s)$, so $\mid r^{\prime}(s)-$ $\exp (s A) r^{\prime}(0)|\leqslant \xi| r^{\prime}(0) \mid$. Integrating this gives the first inequality. Writing $C=a I+$ $\left(a^{2} / 2\right) A+\cdots$, one computes that $\left\|C^{-1}\right\| \leqslant a^{-1}\left(2-e^{a\|A\|}\right)^{-1}$ provided $e^{a\|A\|}<$ 2. Then the requirement that $C$ be invertible with $\left\|C^{-1}\right\|<2 / a$ is easily satisfied. Two estimates suffice to obtain $K$. First, for $t \in[0, a]$,

$$
\begin{aligned}
\mid r(t) & -r(0)-\left(\int_{0}^{t} \exp (s A) d s\right) C^{-1}(r(a)-r(0)) \mid \\
& \leqslant \xi t\left|r^{\prime}(0)\right|+\left\|\int_{0}^{t} \exp (s A) d s\right\|\left\|C^{-1}\right\| \xi a\left|r^{\prime}(0)\right|<\xi a\left|r^{\prime}(0)\right|\left(1+a e^{a\|A\|}\left\|C^{-1}\right\|\right) .
\end{aligned}
$$


Secondly, $|r(a)-r(0)| \geqslant\left|C r^{\prime}(0)\right|-\xi a\left|r^{\prime}(0)\right|$, so

$$
\left|r^{\prime}(0)\right| \leqslant|r(a)-r(0)|\left(\left\|C^{-1}\right\|^{-1}-a / 2\right) \text {. Q.E.D. }
$$

S. Bochner and D. Montgomery [1] proved that an action of a Lie group $G$ is $C^{1}$ as a map from $G \times M$ to $M$. The kind of estimate given above can be used to show that the convergence of a net of actions implies their convergence in the space of $C^{1}$ maps from $G \times M$ to $M$ with the weak topology. That argument essentially exists in the following proof.

Proof of Theorem B. Let $g_{1}(t), \ldots, g_{k}(t)$ be a family of one-parameter subgroups generating the connected Lie group $G$ of dimension $k$. Let $\alpha$ be an action of $G$ on $\mathbf{R}^{n}$ having the origin as a stationary point with $H^{1}=0$ for its linear part $\gamma$. Let $V=\cap_{g \in G} \operatorname{ker}(\gamma(g)-I)$, and let $W$ be a complement to $V$. I assume that the origin is not stable. There are matrices $A_{1}, \ldots, A_{k}$ such that $\gamma\left(g_{j}(t)\right)=\exp \left(t A_{j}\right)$. Let $a \in(0,1]$ be small enough so that for each $j$, the matrix $C_{j}=\int_{0}^{a} \exp \left(s A_{j}\right) d s$ is invertible with $\left\|C_{j}^{-1}\right\|<2 / a$. One result of the lemma (taking the flow $\varphi_{t} x=$ $\left.\exp \left(t A_{j}\right) x\right)$ is that $\operatorname{ker}\left(\gamma\left(g_{j}(a)\right)-I\right)=\cap_{t \in[0, a]} \operatorname{ker}\left(\gamma\left(g_{j}(t)\right)-I\right)$, so that $V$ is also just $\operatorname{ker}\left(\gamma\left(g_{1}(a)\right)-I\right) \cap \cdots \cap \operatorname{ker}\left(\gamma\left(g_{k}(a)\right)-I\right)$. The proof of Theorem A may then be imitated with the distinguished elements $g_{j}(a)$ taking the place of the elements $g_{j}$ there, and with condition (2) replaced by

$\left(2^{\prime}\right) \beta_{i}(g) x \rightarrow \alpha(g) x$ and $D \beta_{i}(g)_{x} \rightarrow D \alpha(g)_{x}$ uniformly for

$$
(g, x) \in\left(g_{1}[0,1] \cup \cdots \cup g_{k}[0,1]\right) \times B .
$$

Again, for $g \in G, u(g)$ is defined to be $\lim _{i \rightarrow \infty}\left(h_{i}\left(x_{i}\right)\right)^{-1 / 2}\left(\beta_{i}(g) x_{i}-x_{i}\right)$ if this limit exists, and by passing to a subsequence we may assume that $u\left(g_{j}(a)\right)$ exists for $j=1, \ldots, k$. Bochner and Montgomery's result states that each map $\varphi_{t} x=$ $\beta_{i}\left(g_{j}(t)\right) x$ is a $C^{1}$ flow. The lemma then says that for $t \in[0, a]$,

$$
\begin{aligned}
& \mid \beta_{i}\left(g_{j}(t)\right) x_{i}-x_{i}-\left(\int_{0}^{t} \exp \left(s A_{j}\right) d s\right) C_{j}^{-1}\left(\beta_{i}\left(g_{j}(a)\right) x_{i}-x_{i}\right) \mid \\
&<\xi K\left|\beta_{i}\left(g_{j}(a)\right) x_{i}-x_{i}\right|
\end{aligned}
$$

where $\xi$ is the supremum of $\left\|D \beta_{i}\left(g_{j}(s)\right)_{x_{i}}-\exp \left(s A_{j}\right)\right\|$ for $s \in[0,1] . \xi \rightarrow 0$ as $i \rightarrow \infty$, so dividing through by $\left(h_{i}\left(x_{i}\right)\right)^{1 / 2}$ and taking limits, we have that $u\left(g_{j}(t)\right)$ exists and in fact satisfies

$$
u\left(g_{j}(t)\right)=\left(\int_{0}^{t} \exp \left(s A_{j}\right) d s\right) C_{j}^{-1} u\left(g_{j}(a)\right) .
$$

Thus $u\left(g_{j}(t)\right)$ is an analytic function of $t$ on the interval $(0, a)$. By the same argument as in the proof of $A$, the existence of this limit on a set generating $G$ implies that it exists and satisfies $u(g h)=\gamma(g) u(h)+u(g)$ for all $g, h \in G$. By this relation, in turn, the analyticity of $\gamma$ and the analyticity of the functions $u\left(g_{j}(t)\right)$ easily imply that $u$ is analytic. In particular, $u$ is a (continuous) nonzero crossed homomorphism, and the same contradiction is reached as in the proof of $A$. Q.E.D.

These proofs ignore the global topology of $\mathbf{R}^{n}$, and their generalization to actions on arbitrary manifolds is simple. Let $\rho$ be a coordinate map at a stationary point $p$ with $\rho(p)=0 \in \mathbf{R}^{n}$. The action $\alpha$ on $M$ carries down to a "partial action" $\alpha^{\prime}$ on $\mathbf{R}^{n}$ 
given by $\alpha^{\prime}(g) x=\rho \circ \alpha(g) \circ \rho^{-1} x$, which is defined for all $(g, x)$ in some open subset $S$ of $G \times \mathbf{R}^{n}$ containing $G \times\{0\}$. The ball $B$ is then chosen so that $J \times \operatorname{clos}(B) \subseteq S$, where $J=\left\{g_{1}, \ldots, g_{k}\right\}$ in the case of Theorem $A$ and $J=$ $g_{1}[0,1] \cup \cdots \cup g_{k}[0,1]$ in the case of Theorem B. The actions $\beta_{i}$ are chosen as before, but they are further required to be close enough to $\alpha$ that the corresponding partial actions $\beta_{i}^{\prime}$ can be defined on $J \times \operatorname{clos}(B)$. Under these conditions, there is for every $g \in G$ a ball $B_{g}$ around the origin and an integer $I_{g}$ such that $\beta_{i}^{\prime}(g)$ is defined on $B_{g}$ for $i \geqslant I_{g}$. For such is true when $g$ is the identity element, and by (2)-(2') the subset of $G$ for which it is true is closed under right multiplication by elements of $J$. The arguments then proceed as before, with many expressions only making sense for sufficiently large $i$. An amalgamation of the proofs of A and B gives

THEOREM AB. If $G$ is a compactly-generated Lie group and $H^{1}\left(G, M_{p}\right)=0$, then $p$ is stable. Furthermore, if $p$ lies in a submanifold $L$ transverse to $V=$ $\cap_{g \in G} \operatorname{ker}\left(D \alpha(g)_{p}-I\right) \subseteq M_{p}$, then actions near $\alpha$ have a stationary point near $p$ in $L$.

As shown by Hirsch [5], linear actions for which $H^{1}$ is not trivial can be perturbed into actions having no stationary point at all. We then have

COROLlary. If $G$ is a compactly-generated Lie group, then triviality of $H^{1}$ is an open condition in the space of linear actions of $G$ on a finite-dimensional vector space E.

2. The stationary set. Let $\operatorname{sta}(\alpha)$ denote the set of all stationary points of $\alpha$, and let $V$ be as in Theorem AB.

TheOReM C. If $G$ is a compactly-generated Lie group and $H^{1}\left(G, M_{p}\right)=0$, then near $p, \operatorname{sta}(\alpha)$ is a $C^{1}$ submanifold which is stable under perturbations of $\alpha$. Specifically, there is a neighborhood $U$ of $p$, a neighborhood $N$ of $\alpha$, a disk $Q$ diffeomorphic to Euclidean space, and for each $\beta \in N$ a closed embedding $q_{\beta} \in$ $C^{1}(Q, U)$ such that (a) $q_{\beta}(Q)=U \cap \operatorname{sta}(\beta)$, and (b) $q_{\beta}$ depends continuously upon $\beta$ in the weak topology on $C^{1}(Q, M)$. Also, the tangent space to $\operatorname{sta}(\alpha)$ at $p$ is $V$.

Proof. Choose a smooth metric on $M$ and norms on $V$ and $\mathbf{R}^{k}$, where $k$ is the codimension of $V$. A map $\Lambda: V \times \mathbf{R}^{k} \rightarrow M$ which is a diffeomorphism onto its image may be thought of as a local product foliation with leaves $L_{v}=$ $\Lambda\left(\{v\} \times \mathbf{R}^{k}\right)$ parameterized by $V$. Let $\Lambda$ be such a map satisfying

(1) $\Lambda(0,0)=p$ with $\Lambda(V \times\{0\})$ tangent to $V$ and

(2) there is a continuous family $\left\{f_{v}\right\}_{v \in V}$ of diffeomorphisms of $M$ such that $f_{0}=\mathrm{id}_{M}$ and $f_{v}\left(L_{0}\right)=L_{v}$ for $v \in V$.

Every vector in $M_{p}$ not tangent to $\Lambda(V \times\{0\})$ is moved by $D \alpha(g)_{p}$ for some $g \in G$. Then by a simple application of the first lemma, there are neighborhoods $N_{0}$ of $\alpha$ and $Q_{0} \times R_{0}$ of the origin in $V \times \mathbf{R}^{k}$ such that if $\beta \in N_{0}$ has stationary points $\Lambda\left(a_{i}, b_{i}\right)$ with $a_{i} \in Q_{0}$ and $b_{i} \in R_{0}$ for $i=1,2$, then $\left|b_{1}-b_{2}\right|<\left|a_{1}-a_{2}\right|$. By shrinking these neighborhoods if necessary, it may also be assumed that for a 
stationary point $\Lambda(a, b)$ of $\beta \in N_{0}$ with $a \in Q_{0}$ and $b \in R_{0}$, the space $\cap_{g \in G} \operatorname{ker}\left(D \beta(g)_{\Lambda(a, b)}-I\right)$ has dimension not greater than that of $V$. In local coordinates, this is a Grassmannian analogue of the infimum of a family of upper semicontinuous functions being upper semicontinuous. Since triviality of $H^{1}$ is an open condition, it may also be assumed that $H^{1}=0$ at such a point.

As $\beta \rightarrow \alpha$ and $v \rightarrow 0$ independently, the action $\beta_{v}$ defined by $\beta_{v}(g) x=$ $f_{v}^{-1} \circ \beta(g) \circ f_{v} x$ tends to $\alpha$. A stationary point of $\beta_{v}$ in $L_{0}$ corresponds by $f_{v}$ to a stationary point of $\beta$ in $L_{v}$. By Theorem $\mathrm{AB}$, there are therefore smaller neighborhoods $N \subseteq N_{0}$ of $\alpha$ and $Q \times R \subseteq Q_{0} \times R_{0}$ of the origin in $V \times \mathbf{R}^{k}$ such that if $\beta \in N$ and $v \in Q$, then $\beta$ has a stationary point in $(\{v\} \times R) \subseteq L_{v}$. The choice of $N_{0}, Q_{0}$ and $R_{0}$ guarantee the uniqueness of this point, which we denote by $q_{\beta} v$. This choice also insures that on compact sets the family $\left\{q_{\beta}\right\}_{\beta \in N}$ is uniformly Lipschitz. Since every stationary point $q_{\beta} v$ is stable, this Lipschitz condition implies that $q_{\beta} v$ is continuous in $\beta$ for fixed $v$. Therefore, by the Ascoli theorem, $q_{\beta}$ varies continuously with $\beta$ in the weak $C^{0}$ topology. With $U=\Lambda(Q \times R)$, assertion (a) is established.

Let $Y(\beta, v)$ be the space of tangent vectors to $M$ at $q_{\beta} v$ which are fixed by the linear action of $\beta . Y$ is upper semicontinuous. That is, given a neighborhood of $Y_{0}=Y\left(\beta_{0}, v_{0}\right)$ in the Grassmannian bundle of $\operatorname{dim}\left(Y_{0}\right)$-planes over $M, Y(\beta, v)$ is contained in a plane of this neighborhood if $(\beta, v)$ is near $\left(\beta_{0}, v_{0}\right)$. Let $A(\beta, v)$ denote the set of tangent vectors to $M$ at $q_{\beta} v$ which can be represented by a curve $r(t)$ with $r(0)=q_{\beta} v$ and $r\left(t_{i}\right) \in \operatorname{sta}(\beta)$ for some sequence $t_{1}, t_{2}, \ldots$ of positive numbers tending to zero. Clearly $A(\beta, v) \subseteq Y(\beta, v)$. Also, by assumption, the dimension of $Y(\beta, v)$ is not greater than that of $V$. On the other hand, by the Lipschitz condition, if one takes any curve $a(t)$ in $V$ with $a(0)=v$, there is a curve $b(t)$ in $\mathbf{R}^{k}$ such that $r(t)=(a(t), b(t))$ is in $A(\beta, v)$. This says that $D \Lambda^{-1}(A(\beta, v))$ projects onto $V$ along $\mathbf{R}^{k}$. Thus the dimensions of $Y(\beta, v)$ and $V$ are equal, $A(\beta, v)=Y(\beta, v)$ and $q_{\beta}$ is differentiable at $v$ with $D q_{\beta}\left(V_{v}\right)=Y(\beta, v)$. The upper semicontinuity of $Y$ is therefore continuity, and $q_{\beta}$ varies continuously with $\beta$ in the weak $C^{1}$ topology. Q.E.D.

3. Applications to certain classes of groups. For a particular group, triviality of $\boldsymbol{H}^{\mathbf{1}}$ might be expressed as a more tangible condition on linear actions. For example, $H^{1}$ is trivial for a linear action of $\mathbf{Z}$ precisely when 1 is not an eigenvalue of the generating linear transformation. On the other hand, this condition is not well understood for solvable groups. Let $G$ be a group such that every short exact sequence $0 \rightarrow E \rightarrow F \rightarrow \mathbf{R} \rightarrow 0$ of (finite-dimensional) $G$-modules splits. This is the case, for example, when $G$ is compact or is a semisimple analytic group. Then by a trick of J. Milnor (Lemma 2.3 of [6]), $H^{1}$ is trivial for every linear action $\gamma$ of $G$. The trick is the observation that an affine action $\gamma_{u}$ on $E$ corresponds to a linear action $(g ; x, t) \mapsto(\gamma(g) x+t u(g), t)$ on $E \times \mathbf{R}$. This gives

Corollary of Theorem C. Let $G$ be a semisimple analytic group acting on a compact manifold by $\alpha$. Then $\operatorname{sta}(\alpha)$ is a $C^{1}$ submanifold of $M$. Let $i_{\alpha}$ denote the inclusion of $\operatorname{sta}(\alpha)$ into $M$. If $\beta$ is near $\alpha$, then there is a diffeomorphism $f$ from $\operatorname{sta}(\alpha)$ onto $\operatorname{sta}(\beta)$ with $i_{\beta} \circ$ f near $i_{\alpha}$ in $C^{1}(\operatorname{sta}(\alpha), M)$. 
This confirms a conjecture of Hirsch [4]. In [2], V. Guillemin and S. Sternberg showed that an analytic action of a semisimple analytic group can be linearized near a stationary point. Since any linear representation of a semisimple analytic group is equivalent to all representations near it, it follows that such an action is analytically structurally stable near that point. In the same paper, an example is given which shows that similar statements do not carry over to the $C^{\infty}$ case.

A second example, whose proof is left to the reader, is suggested by Theorem 3 of [9].

COROLlary. Let $G$ be a compactly-generated Lie group and $M$ a connected manifold of dimension at least one. Then the trivial action of $G$ on $M$ is isolated if and only if $G$ admits no nontrivial continuous homomorphism into $\mathbf{R}$.

Hirsch has shown [3] that for any linear action $\gamma$ of a nilpotent group, $H^{1}$ will be trivial provided $\operatorname{sta}(\gamma)=\{0\}$.

THEOREM D. Let $G$ be a nilpotent topological group which is either compactly-generated or generated by arbitrarily small neighborhoods of its identity element $e$. A stationary point $p$ of an action $\alpha$ is stable provided the linear action $\gamma$ on $M_{p}$ fixes only the origin.

Proof. There are finitely many elements $g_{1}, \ldots, g_{k}$ of $G$ (in fact, $k<n$ ) such that $\operatorname{ker}\left(\gamma\left(g_{1}\right)-I\right) \cap \cdots \cap \operatorname{ker}\left(\gamma\left(g_{k}\right)-I\right)=0$. We place the discrete topology on the subgroup $H$ they generate. Since compact, i.e. finite, subsets of $H$ are compact in $G$, restriction to $H$ is a continuous map from $G$-actions to $H$-actions. Therefore, by Hirsch's result and Theorem $\mathrm{C}$, there are neighborhoods $U$ of $p$ and $N$ of $\alpha$ such that if $\beta \in N$, then $\beta\left(g_{1}\right), \ldots, \beta\left(g_{k}\right)$ have a unique common fixed point $p_{\beta}$ in $U$. Furthermore, $p_{\beta} \rightarrow p$ as $\beta \rightarrow \alpha$. If $g, h \in G$ and if $q \in M$ is fixed by $\beta(g)$ and $\beta\left(h^{-1} g h g^{-1}\right)$, then $\beta(h) q$ is also fixed by $\beta(g)$, since $\beta(g) \beta(h) q=$ $\beta(h) \beta\left(h^{-1} g h g^{-1}\right) \beta(g) q=\beta(h) q$. Thus if $q$ is an isolated fixed point of $\beta(g)$ and $\beta(h)$ does not move $q$ very much, then $\beta(h) q$ must equal $q$. This kind of argument is made in two cases.

Case $1 . G$ is generated by a compact set $S$. Let $S_{0}=S$, and for $i \geqslant 1$ let $S_{i}=\left\{h^{-1} g_{j} h g_{j}^{-1}: h \in S_{i-1}\right.$ and $\left.1 \leqslant j \leqslant k\right\}$. Since $G$ is nilpotent, there is some integer $v$ for which $S_{v}=\{e\}$. Let $N^{\prime} \subseteq N$ be such that if $\beta \in N^{\prime}$, then $\beta\left(S_{0} \cup \cdots \cup S_{v}\right) p_{\beta} \subseteq U$. By inducting downward on $n$, one establishes that $\beta\left(S_{n}\right) p_{\beta}=p_{\beta}$ for $v \geqslant n \geqslant 0$. The result is that $p_{\beta}$ is fixed by $\beta(g)$ whenever $g \in S_{0}=S$, hence for all $g \in G$. This kind of argument is used by Hirsch in [4].

Case 2. $G$ is generated by arbitrarily small neighborhoods of $e$. For any $\beta \in N$, let $A$ be a neighborhood of $e$ such that $\beta(A) p_{\beta} \subseteq U$. There is a smaller neighborhood $B$ of $e$ such that if one defines $B_{0}=B$ and $B_{i}=\left\{h^{-1} g_{j} h g_{j}^{-1}: h \in B_{i-1}\right.$ and $1<j \leqslant k\}$ for $i \geqslant 1$, then $B_{0} \cup \cdots \cup B_{v} \subseteq A$. By the same argument as in Case $1, p_{\beta}$ is fixed by $\beta(g)$ whenever $g \in B_{0}=B$, hence for all $g \in G$. Q.E.D.

\section{BIBLIOGRAPHY}

1. S. Bochner and D. Montgomery, Groups of differentiable and real or complex analytic transformations, Ann. of Math. (2) 46 (1945), 685-694. 
2. V. Guillemin and S. Sternberg, Remarks on a paper of Hermann, Trans. Amer. Math. Soc. 130 (1968), 110-116.

3. M. Hirsch, Flat manifolds and the cohomology of groups, Algebraic and Geometric Topology, Lecture Notes in Math., vol. 664, Springer-Verlag, Berlin and New York, 1977.

4. Stability of stationary points of group actions, Bifurcation Theory and Applications in Scientific Disciplines, edited by O. Gurel and H. Rössler, Ann. New York Acad. Sci. 316 (1979), 43-48. 5. _ Stability of stationary points and the cohomology of groups, Proc. Amer. Math. Soc. (to appear).

6. J. Milnor, On fundamental groups of complete affinely flat manifolds, Advances in Math. 25 (1977), 178-187.

7. R. Palais, Equivalence of nearby differentiable actions of a compart group, Bull. Amer. Math. Soc. 67 (1961), 362-364.

8. J. D. Stasheff, Continuous cohomology of groups and classifying spaces, Bull. Amer. Math. Soc. 84 (1978), 513-530.

9. W. Thurston, A generalization of the Reeb stability theorem, Topology 13 (1974), 347-352.

2740 Russell Street, Berkeley, California 94705 